\title{
Cintigrama renal D MSA en niños con primera pielonefritis aguda: correlación con exámenes de laboratorio, ecografía y la presencia de reflujo vésico ureteral
}

Gilda Donoso $\mathbf{R}^{1}$, Gabriel Lobo $\mathbf{S}^{\mathbf{1}}$, Francisca Arnello $\mathbf{V}^{\mathbf{1}}$, María Paz Arteaga $V^{1}$, Pilar Hevia J2 ${ }^{2}$, Pía Rosati $M^{3}$, Elizabeth Lagos $R^{3}$, Carlos Wolff $F^{4 a}$, Andrés Pérez $R^{1 b}$, César Jiménez $\mathrm{J}^{1 \mathrm{~b}}$.

\section{Tc 99M DMSA scintigraphy in children with a first episode of pyelonephritis}

Background: Tc99m DMSA (dimercaptosuccinic acid) scintigraphy has a high sensitivity for the detection of cortical kidney damage. Aim: To evaluate the Tc99m DMSA renal scintigraphy in children with a first episode of acute pyelonephritis and its association with laboratory parameters, kidney ultrasound and vesicoureteral reflux. Patients and methods: We studied 143 children (age range 8 days, 12 years, 66\% female) hospitalized with the clinical diagnosis of acute pyelonephritis (first episode) with a positive urine culture and a renal scintigraphy performed within seven days of diagnosis. DMSA was considered the gold standard for the detection of cortical lesions. Its results were related to the presence of fever, C-reactive protein (CRP), erythrocyte sedimentation rate (VHS), white blood count (WBC), ultrasound examination and vesicoureteral reflux. Results: Seventy nine percent of the population had an abnormal DMSA scan. There were no differences between sex, age and laboratory parameters in children with normal or abnormal DMSA scans, except for CRP ( $p<0.005)$. Ultrasound was coincident with the scan in $32 \%$ of patients. Eighteen percent had vesicoureteral reflux. Conclusions: There is a high proportion of abnormal DMSA scans in children with a first episode of acute pyelonephritis (Rev Méd Chile 2004; 132: 58-64).

(Key Words: Pyelonephritis; Radionuclide imaging; Technetium Tc 99 dimercaptosuccinic acid)

Recibido el 19 de marzo, 2003. Aceptado en versión corregida el 10 de noviembre, 2003.

${ }^{1}$ Servicio de Medicina Nuclear, Hospital San Juan de Dios, Universidad de Chile. ${ }^{2}$ Departamento de Pediatría Occidente, Universidad de Chile. ${ }^{3}$ Servicio de Nefrología Infantil, Hospital San Juan de Dios. ${ }^{4}$ Departamento de Medicina Occidente, Universidad de Chile. aBioquímico

bTecnólogo Médico

Correspondencia a: Dra. Gilda Donoso R. Servicio de Medicina Nuclear. CDT - Hospital San Juan de Dios. Avenida Diego Portales 3239, Santiago. Fono: 4506869.

E-mail: gdonosor @yahoo.com 
L a infección del tracto urinario (ITU) es una de las enfermedades infecciosas más común en niños. Puede estar limitada a la vejiga, cistitis o comprometer el parénquima renal, pielonefritis $\mathrm{y}$, en este caso, existe la posibilidad de daño renal irreversible, cicatrices, con secuelas de hipertensión arterial y falla renal crónica. Según un estudio realizado en nuestro país, en $17 \%$ de los niños en programa de diálisis o trasplante, la causal de insuficiencia renal fue pielonefritis crónica secundaria a reflujo vésico ureteral o infección urinaria ${ }^{1}$.

El diagnóstico de ITU se basa en urocultivo positivo, con recuentos adecuados para cada técnica de recolección de orina ${ }^{2}$, y para diferenciar si una ITU es alta o baja existen diferentes marcadores clínicos y de laboratorio: fiebre alta, leucocitosis, aumento de la proteína $\mathrm{C}$ reactiva (PCR), aumento de la velocidad hemática de sedimentación (VHS) y para algunos, cintigrama renal estático con DMSA (ácido dimercapto-succínico) positivo. El diagnóstico clínico de pielonefritis aguda se basa habitualmente en los criterios de $\mathrm{Jodal}^{3}$, que se ven igualmente comprometidos en presencia de cualquier cuadro infeccioso, y es particularmente difícil diferenciar si una ITU es alta o baja en lactantes, especialmente menores de 1 año.

La detección de lesiones corticales renales por medio de radioisótopos es una de las técnicas más usadas en el campo de la medicina nuclear pediátrica. El cintigrama renal con DMSA (CR DMSA) marcado con tecnecio 99m (Tc 99m) es considerado un examen de elección para la determinación de masa tubular funcionante, ya que este trazador se acumula en las células tubulares renales proximales en relación a la función regional, siendo muy sensible para la detección de daño regional cortical, lo que ha sido demostrado y validado en numerosos estudios clínicos y experimentales ${ }^{4-7}$.

Existe controversia sobre la utilidad del cintigrama renal en la fase aguda de la ITU existiendo sí, amplio consenso en su uso sistemático para la detección de cicatrices ${ }^{8}$.

En nuestro estudio, quisimos evaluar los hallazgos del CR DMSA realizado en niños hospitalizados con diagnóstico presuntivo de primera pielonefritis aguda (PNA), durante la fase aguda de la enfermedad y su relación con los exámenes de laboratorio habitual, ecografía y estudio de reflujo vésico ureteral (RVU).

\section{MATERIAL Y MÉTODO}

Este estudio fue realizado en niños hospitalizados en el Servicio de Pediatría del Hospital San Juan de Dios referidos a Medicina Nuclear con el diagnóstico presuntivo de primera pielonefritis aguda. Se incluyeron 143 niños, de ellos 94 eran niñas (66\%), con un promedio de edad de 14 meses (rango: 8 días-12 años). El 79\% tenía menos de 2 años.

El tiempo medio entre el inicio de los síntomas y el diagnóstico fue de 5 días (intervalo 1-21 días). Todos los cintigramas fueron realizados dentro de los 7 días del diagnóstico clínico de PNA, el que fue realizado considerando fiebre, compromiso del estado general, anorexia, náuseas, vómitos, sedimento de orina alterado y urocultivo positivo (más de 100.000 colonias por $\mathrm{ml}$, por recolector o por orina de $2^{\circ}$ chorro; más de 10.000 por sondeo vesical o 1 o más colonias por punción vesical); leucocitosis, más de 15.000 glóbulos blancos $/ \mathrm{mm}^{3}$, aumento de la VHS, más de $30 \mathrm{~mm} / \mathrm{h}, 0$ aumento de la PCR, más de $20 \mathrm{mg} / \mathrm{dl}$.

Los estudios por imágenes efectuados, además del CR DMSA, fueron ecografía renal, uretrocistografía radiológica o cistografía isotópica.

Se excluyeron pacientes con malformaciones congénitas, excepto RVU y doble sistema pielocalicilar.

El cintigrama renal fue realizado con DMSA-Tc $99 \mathrm{~m}$. Las dosis usadas fueron calculadas según la edad. Dos a tres horas después de la inyección iv del trazador se obtuvieron 4 imágenes en diferentes proyecciones (póstero anterior, oblicua posterior izquierda, oblicua posterior derecha y ántero posterior) con gammacámara Picker Dyna unida a computador alfa nuclear donde se realizó el cálculo de la función renal diferencial.

El CR DMSA fue considerado el gold standard para la detección de lesiones corticales y se catalogó como anormal cuando se encontró una o más áreas de menor captación focal cortical del trazador, disminución difusa de captación, alteración del tamaño renal o menor función relati$\mathrm{va}^{5,8,9}$, no siendo ninguno de estos parámetros específicos de PNA (Figuras 1 y 2) 5 ,8,9.

La ecografía fue descrita como normal, pieloectasias, alteraciones de la ecogenicidad, nefropatía médica, nefromegalia, doble sistema excretor ${ }^{10}$. El $94 \%$ de ellas fue realizada dentro 


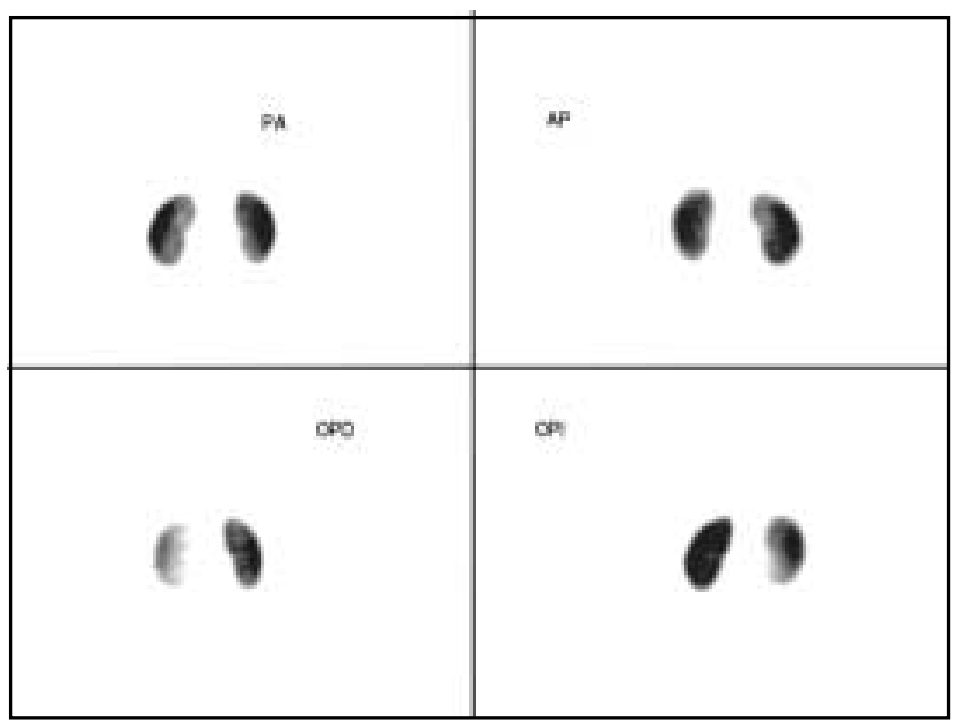

FIgURA 1. Cintigrama renal DMSA en límites normales. Distribución homogénea del trazador en ambos riñones. PA: póstero-anterior; AP: ántero-posterior; OPD: oblicua posterior derecha; OPI: oblicua posterior izquierda.

FIGURA 2. Cintigrama renal DMSA alterado. Menor contraste de ambos polos de riñón izquierdo. PA: póstero-anterior; AP: ántero-posterior; OPD: oblicua posterior derecha; OPI: oblicua posterior izquierda.

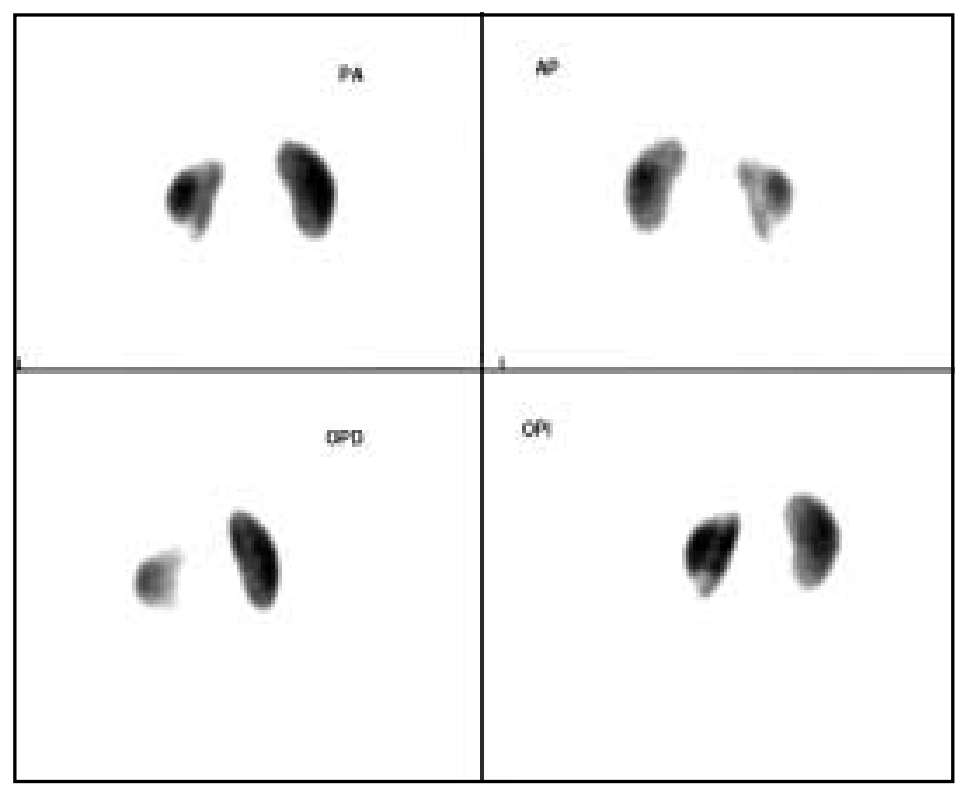

del primer mes del diagnóstico de PNA, siendo la mayoría realizada en la primera semana $(78,7 \%)$. Se considera coincidente la ecografía con el CR DMSA cuando ésta informa alteración en el mismo lugar de alteración descrita en el CR.

El 68,7\% de los estudios de RVU se efectuaron dentro del primer mes del diagnóstico de PNA.

Los datos obtenidos de fiebre, PCR, leucocitosis, VHS, ecografía y estudio de RVU se correlacio- naron con los resultados del CR DMSA (normal o anormal), realizando una comparación entre los diferentes grupos.

Para el análisis estadístico se aplicó pruebas de Mann Whitney, McNemar y Chi cuadrado, considerándose diferencia significativa un $\mathrm{p}<0,005 \mathrm{y}$ test $\mathrm{Z}$ para dos proporciones, considerando significativo un $\mathrm{p}<0,05$. 
Tabla 1. Resultados obtenidos en los diferentes parámetros de laboratorio estudiados según CR D M SA

\begin{tabular}{|lccc|}
\hline & $\begin{array}{c}\text { CR Normal } \\
\text { promedio } \pm \text { DS) }\end{array}$ & $\begin{array}{c}\text { CR Alterado } \\
\text { (promedio } \pm \text { DS) }\end{array}$ & Valor $\mathrm{p}$ \\
\hline Fiebre & $39,04 \pm 0,71$ & $39,33 \pm 0,75$ & 0,060 \\
PCR & $54,1 \pm 43,83$ & $137,61 \pm 89,68$ & $0,001 *$ \\
VHS & $56,92 \pm 52,33$ & $76,98 \pm 33,98$ & 0,009 \\
Leucocitos & $16.063 \pm 58,61$ & $20.592 \pm 9.258 .9$ & 0,017 \\
\hline
\end{tabular}

*Estadísticamente significativo (test de Mann Whitney)

\section{RESUlTADOs}

El 79\% de la población (113) tuvo un CR DMSA anormal, y de éstos, $68 \%$ correspondió a mujeres (77). Entre los 30 pacientes con CR DMSA normales, 57\% correspondió a mujeres (17) p =0,337.

Según edad, se encontró que de los 30 pacientes mayores de 2 años, 90\% tenía un CR DMSA alterado, y de los 113 niños menores de 2 años, 76\% tenía CR DMSA alterado (86), $p=0,159$.

Las diferencias encontradas no son estadísticamente significativas.

Las alteraciones cintigráficas más frecuentes fueron lesiones de tipo focal en $46 \%$ y unilateral, en $65 \%$. Sólo $13 \%$ tuvo función relativa disminuida.

Laboratorio: En la Tabla 1 se muestra el resumen de los promedios y desviación estándar (DS) de los resultados de laboratorio y fiebre, separados según resultado del CR DMSA.

Se observó que el único parámetro que presentó diferencia significativa entre el grupo con CR DMSA normal y el grupo con CR DMSA alterado fue la PCR que presentó valores mayores en el grupo de niños con CR DMSA alterado.

Ecografía: Fue realizada en 127 de los 143 niños estudiados (89\%). Se observó coincidencia de esta técnica y CR DMSA en 41 de 127 pacientes (32\%), y no coincidencia en 86 de 127 (68\%) ( $p=<0,001$, prueba de McNemar) (Tabla 2).

La Tabla 3 muestra la sensibilidad (S), especificidad (E), valor predictivo positivo (VPP) y valor predictivo negativo (VPN) de los diferentes parámetros de laboratorio y de la ecografía en la detección de daño renal cortical evidenciado por el CR DMSA.
Tabla 2. Ecografía y cintigrama renal en niños con primera pielonefritis aguda

\begin{tabular}{|lccc|}
\hline & $\begin{array}{c}\text { Ecografía } \\
\text { alterada }\end{array}$ & $\begin{array}{c}\text { Ecografía } \\
\text { normal }\end{array}$ & Total \\
\hline CR DMSA alterado & $19^{*}$ & 81 & 100 \\
CR DMSA normal & 5 & $22^{*}$ & 27 \\
\hline
\end{tabular}

*Exámenes concordantes. (Prueba de Mac Nemar, $\mathrm{p}=0,001)$.

Tabla 3. Rendimiento relativo de diferentes parámetros estudiados

\begin{tabular}{|lcccc|}
\hline & $\begin{array}{c}\text { PCR } \\
>20 \mathrm{mg} / \mathrm{dl}\end{array}$ & $\begin{array}{c}\text { VHS } \\
>30 \mathrm{~mm} / \mathrm{h}\end{array}$ & $\begin{array}{c}\text { Leucocitos } \\
>15.000 / \mathrm{mm}^{3}\end{array}$ & Ecografía \\
\hline S & $85 \%$ & $84 \%$ & $82 \%$ & $23 \%$ \\
E & $40 \%$ & $29 \%$ & $27 \%$ & $78 \%$ \\
VPP & $92 \%$ & $89 \%$ & $71 \%$ & $70 \%$ \\
VPN & $24 \%$ & $21 \%$ & $40 \%$ & $36 \%$ \\
\hline
\end{tabular}

Diferencias estadísticamente significativas (test Z para 2 proporciones):

- PCR, VHS y leucocitos son más sensibles que la ecografía.

- PCR es más específica que la VHS y leucocitos.

- La ecografía es más específica que la PCR, VHS y leucocitos.

- PCR tiene mayor VPP que leucocitos y ecografía.

- VHS tiene mayor VPP que leucocitos y ecografía.

- Ecografía y leucocitos tienen mayor VPN que la PCR y VHS. 
Tabla 4. Relación entre el cintigrama renal y la presencia de reflujo vesicoureteral en niños con primera pielonefritis aguda

\begin{tabular}{|lccr|}
\hline & $\begin{array}{c}\text { CR DMSA } \\
\text { normal }\end{array}$ & $\begin{array}{c}\text { CR DMSA } \\
\text { anormal }\end{array}$ & Total \\
\hline RVU negativo & 28 & 76 & 104 \\
RVU positivo & 1 & 22 & 23 \\
Total & 29 & 98 & 127 \\
\hline
\end{tabular}

Prueba de Chi cuadrado: $p=0,039$.

Uretrocistografía o cistografía isotópica: Se demostró RVU en 23 de 127 niños (18\%). De los pacientes con CR DMSA normal, sólo 1 se asoció a RVU leve, en cambio de los 98 pacientes con CR DMSA anormal, 22 de ellos presentaron RVU. Tabla 4 .

$\mathrm{Al}$ analizar la frecuencia de positividad del RVU entre el grupo con CR DMSA normal y CR DMSA alterado, no se encontraron diferencias significativas $(p=0,039)$ (prueba de Chi cuadrado).

\section{DiscUSIÓN}

De acuerdo a un reciente trabajo de consenso, la primera indicación del CR DMSA es la evaluación de la presencia de cicatriz renal, al menos 6 meses después de un episodio de ITU. Su uso rutinario durante la fase aguda de una pielonefritis no es aceptado por todos ${ }^{8}$.

En este estudio se evaluaron los hallazgos del CR DMSA en niños, durante la fase aguda de una pielonefritis diagnosticada clínicamente, el valor diagnóstico de parámetros de laboratorio, clínicos, el ultrasonido y la presencia de RVU. Además se estudió la relación de éstos con el CR DMSA, considerado como el gold standard en la detección de daño cortical, avalados en la evidencia bibliográfica de su sensibilidad cercana al 100\% ${ }^{4-6}$.

En nuestra población obtuvimos $79 \%$ de positividad para el CR DMSA. El 21\% restante, que tenía CR DMSA normal, con parámetros clínicos y de laboratorio sugerentes de PNA, se puede explicar por un compromiso infeccioso extracortical, pielitis o ureteritis, o por lesiones corticales muy pequeñas que no fueron detectadas por las técnicas cintigráficas planares convencionales. Estudios experimen-
Tabla 5. Relación entre cintigrama renal y severidad del RVU en niños con primera pielonefritis aguda

\begin{tabular}{|lcrr|}
\hline & $\begin{array}{c}\text { CR DMSA } \\
\text { normsal }\end{array}$ & $\begin{array}{c}\text { CR DMSA } \\
\text { anormal }\end{array}$ & Total \\
\hline RVU grados I-II & 1 & 13 & 14 \\
RVU grados III-IV-V & 0 & 9 & 9 \\
Total & 1 & 22 & 23 \\
\hline
\end{tabular}

tales señalan que lesiones pequeñas, que comprometen sólo 5 a 10\% del parénquima, son difíciles de detectar con cintigrafía. Esta sensibilidad podría ser aumentada con exámenes tomográficos (single photon emission computarized, SPECT), pero son necesarios más estudios al respecto ${ }^{11-13}$. Un CR DMSA negativo también puede deberse a que el niño esté cursando con más de un cuadro infeccioso a la vez, lo que falsea el valor del score clínico para diagnosticar pielonefritis aguda.

La mayoría de los pacientes incluidos en este estudio era menor de 2 años, lo que puede ser explicado a que eran sólo pacientes hospitalizados, lo que ocurre con mayor frecuencia entre los niños más pequeños, para el mejor manejo de las condiciones generales y el inicio del tratamiento antibiótico intravenoso.

En nuestra casuística, la presencia o ausencia de daño renal cortical no se relacionó con la edad ni con el sexo, lo que apoya la conducta que todo niño con ITU debe ser estudiado independientemente de estos factores.

Las lesiones de tipo focal fueron las más frecuentemente encontradas en nuestra población y el patrón cintigráfico de disminución difusa de captación, con mayor tamaño renal, que sería propio de la fase aguda, fue poco frecuente, hallazgos concordantes con lo descrito en la literatura ${ }^{5,9}$. La cantidad de riñones disminuidos de tamaño encontrados entre nuestros pacientes (13\%), que no es característico de la fase aguda, podría ser explicado porque es posible que estos niños hayan tenido uno o más episodios de pielonefritis aguda previos a nuestra evaluación, sin haber sido diagnosticados ni tratados, y que dejaron cicatrices como secuelas, también puede deberse a riñones congénitamente hipoplásicos, elementos que no fueron analizados en esta oportunidad. 
Entre los parámetros de laboratorio analizados, sólo se encontraron diferencias significativas en la PCR, que fue mayor en los pacientes con CR DMSA alterado, lo que ya ha sido descrito por otros autores ${ }^{14}$.

Al realizar el cálculo de sensibilidad, especificidad y valor predictivo positivo y negativo, vemos que en general, todos los parámetros de laboratorio poseen una alta sensibilidad, mayor que la ecografía, pero tienen una baja especificidad para detectar daño renal cortical, siendo la ecografía significativamente más específica. Esto podría ser diferente si se cambian los puntos de corte para definir la anormalidad de estos exámenes. En este estudio, los puntos de corte elegidos para catalogar de anormales la VHS, PCR y recuento de leucocitos fueron los que se usan habitualmente en la clínica. La PCR y VHS poseen la mayor sensibilidad y VPP, pero, al igual que los otros, una muy baja especificidad y bajo VPN; dicho de otra manera, con valores de PCR mayores de 20 unidades, de VHS mayor de $30 \mathrm{y}$ leucocitos mayor de 15.000, según este estudio, sería posible predecir el compromiso cortical renal, pero si los valores son bajos, no podemos descartarlo, siempre y cuando exista la certeza que no hay otra patología infecciosa asociada, la cual pueda causar elevación de los valores de PCR.

En la estrategia de identificar precozmente niños de riesgo para desarrollar cicatrices, es de gran valor la detección de compromiso renal en los pacientes con infección urinaria durante la fase aguda, lo que puede influir en la decisión de un tratamiento más agresivo o prolongado, lo que disminuiría el riesgo de dejar secuelas o puede influir también en la decisión de seguimiento y vigilancia de estos niños o la necesidad de profilaxis antibiótica. Por todo esto, es importante contar con un examen de alta sensibilidad y especificidad como lo es el CR DMSA.

La ecografía en nuestros pacientes mostró una muy baja sensibilidad en la detección de daño renal cortical agudo, con una concordancia con el CR DMSA sólo de 32\%, diferencia estadísticamente significativa, lo que se explica porque la información que entrega cada una de estas técnicas es diferente. La especificidad de la ecografía es mejor que las otras técnicas, pero sólo de 78\%. El rol fundamental de la ecografía en el estudio de niños en la fase aguda de una ITU, estaría en la caracterización de lesiones vistas en el CR DMSA, para excluir quistes o tumores, por ejemplo, o la detección de malformaciones asociadas. En etapas tardías de PNA, la ecografía es capaz de evidenciar cicatrices renales ya establecidas ${ }^{15,16}$.

$\mathrm{Al}$ analizar la comparación entre CR DMSA alterado y la presencia de RVU, encontramos que $18 \%$ de los casos tenía RVU positivo, cifras similares a las descritas por otros autores e informada por nosotros en una comunicación previa ${ }^{17}$. En pacientes con CR DMSA normal, el RVU estuvo presente sólo en 3\% de los casos. A pesar de una tendencia a que la presencia de RVU es más importante en los pacientes con CR DMSA alterado, estas diferencias no fueron estadísticamente significativas, lo que podría explicarse porque es una población muy pequeña. Otro factor importante a considerar es que el RVU en el paciente con CR DMSA normal fue de grado leve.

De los pacientes con RVU positivo, 39\% tenía RVU grados III, IV ó V y el 61\% restante eran RVU I ó II, observaciones ya descritas en otras publicaciones ${ }^{18-21}$.

Estos resultados podrían sugerir que no sería absolutamente necesario realizar estudios de RVU en niños con PNA y CR DMSA normal, ya que probablemente el estudio será negativo 0 , si es positivo, el reflujo probablemente será de poca trascendencia. Este concepto es discutido en la actualidad y la decisión dependerá de cada caso particular y la experiencia de cada grupo de trabajo. Datos disponibles aseguran que el riesgo de desarrollar cicatrices es cero cuando el CR DMSA en la fase aguda de la ITU es normal y aproximadamente 35\% cuando existen alteraciones ${ }^{22-24}$.

Debido a la importancia de la detección de daño cortical renal en una ITU para el pronóstico y seguimiento de estos pacientes, creemos que es importante la realización del CR DMSA en la fase aguda, a pesar de que hay oportunidades en que no es posible definir si la lesión encontrada es aguda 0 crónica.

En conclusión, los parámetros clínicos y de laboratorio no son capaces de diferenciar una ITU con o sin compromiso cortical renal, elemento importante a considerar ya que es un indicador de riesgo de desarrollar cicatriz renal, independientemente de la presencia o ausencia de RVU. Por todo esto, recomendamos el uso del CR DMSA en el estudio inicial de niños con ITU. 


\section{REFERENCIAS}

1. Lagomarsino E, Valenzuela A, Cavagnaro F, Solar E. Chronic renal failure in pediatrics 1996. Chilean survey. Pediatr Nephrol 1999; 13: 288-91.

2. American Academy of Pediatrics. Committee on Quality Improvement. Subcommittee on Urinary Tract Infection. Practice parameter: the diagnosis, treatment and evaluation of the initial urinary tract infection in febrile infants and young children. Pediatrics 1999; 103: 843-52.

3. Jodal U, Lindberg U, Lincoln K. Level diagnosis of symptomatic urinary tract infections in childhood. Acta Paediatr Scand 1975; 64: 201-8.

4. Parkhouse HF, Godley ML, Cooper J, Ridson RA, RANSLEY PG. Renal imaging with Tc $99 \mathrm{~m}$ labelles DMSA in the detection of acute pyelonephritis: an experimental study in the pig. Nucl Med Commun 1989; 10: 63-70.

5. Massoud M, Rushton HG. Renal cortical scintigraphy in the diagnosis of acute pyelonephritis. Sem Nucl Med 1992; 2: 98-111.

6. Rushton HG, Majd M, Chandra R, Yim D. Evaluation of Tc 99m-dimercaptosuccinic acid renal scans in experimental acute pyelonephritis in piglets. J Urol 1998; 140: 1169-74.

7. Rushton HG, Majd M. Dimencaptosuccinic acid renal scintigraphy for the evaluation of pyelonephritis and scarring: a review of experimental and clinical studies. J Urol 1992; 148: 1726-32.

8. Piepsz A, Blaufoux MD, Gordon I, Granerus G, Majd M, O'Really $P$ et al. Consensus on renal cortical scintigraphy in children with urinary tract infection. Sem Nucl Med 1999; 2: 160-74.

9. De Sadeleer C, Piepsz A, Hamphrey $H$. Atlas on acute pyelonephritis in children. Clin Nucl Med 2000; 7: 541-5.

10. Roebuck DJ, Howard RG, Matrewew C. How sensitive is ultrasound in the detection of renal scars? Br J Radiol 1999; 72: 345-8.

11. Handinaker H, Young BW, Lawestein JM. Clinical experience with $99 \mathrm{~m}$ Tc DMSA a new renal imaging agent. J Nucl Med 1975; 16: 28-32.

12. Wiшамs D. Renal single photon emission computed tomography: should we do it? Sem Nucl Med 1992; 2: 112-21.

13. Tzu Chen Yen, Kai Yuan Tzan, Wan Yu Lin, Wei Perng Chen, Ching-Yuang Lin. Identification of new renal scarring in repeated episodes of acute pyelonephritis using Tc 99m DMSA renal SPECT. Clin Nucl Med 1998; 12: 828-31.

14. Biggi A, Dardanelu L, Pomero G, Cussino P, Noello $\mathrm{CH}$, Noешо O Eт AL. Acute renal cortical scintigraphy in children with a first urinary tract infection. Pediatr Nephrol 2001; 16: 733-8.

15. Benador D, Benador N, Slosman D, Nissie D, MERMLLD B, GirARDin E. Cortical scintigraphy in the evaluation of renal parenchymal changes in children with pyelonephritis. J Pediatr 1994; 124: 17-20.

16. Rushton HG, Massoud M, Jantausch B, Wiederman B, BeLman. Renal scarring following reflux and nonreflux pyelonephritis in children: evaluation with technetium dimercapto succinic acid scintigraphy. J Urol 1992; Vol 147: 1327-32.

17. Donoso G, Lobo G, Arteaga MP, Arnelo F, Pérez A, JiMÉNEZ C. Cintigrama renal DMSA tc $99 \mathrm{~m}$ y cistografía isotópica directa en niños con infección urinaria recurrente. Alasbimn J 2003; 5: 20.

18. Ditchifeld M, De Campo J, Cook D, Nolan T, Powell H, SLoANe R ET AL. Vesicoureteral reflux: an accurate predictor of acute pyelonephritis in childhood urinary tract infection? Radiology 1994; 190: 413-5.

19. GoRDON I. Vesico ureteric reflux, urinary tract infection and renal damage in children. Lancet 1995; 346: 489-90.

20. Nguyen H, Bauer S, Peters C, Conowy L, Gobert R, BORER J ET AL. 99m Technetium dimercaptosuccinic acid renal scintigraphy abnormalities in infants with sterile high grade vesicoureteral reflux. J Urol 2000; Vol 164: 1674-9.

21. Oreluana $P$, Baquedano P, Prieto $C$, Acuña O, Sierralta P, Cavagnaro F et al. Lesiones parenquimatosas renales y anomalías del tracto urinario en niños con infección urinaria. Rev Chil Radiología 1997; 3: 141-4.

22. JaKobsSON B, SödERLundh S, Berg V. Diagnostic significance of $99 \mathrm{~m}$ Tc- dimercaptosuccinic acid (DMSA) scintigraphy in urinary tract infection. Arch Dis Childhood 1992; 67: 1338-42.

23. JaKobsson B, JaCobson SH, HJalmar K. Vesico ureteric reflux and other risk factors for renal damage: identification of high and low risk children. Acta Paediatr Suppl 1999; 431: 31-9.

24. Oreluana P, Cavagnaro F, Baquedano P, Lagomarsino E, García C, Vimarroel L et al. Factores de riesgo de daño renal permanente en niños con infección del tracto urinario. Rev Méd Chile 2002; 130: 1147-53. 